\title{
Effects of Variable Viscosity and Thermal Conductivity on Combined Free-Forced Convection Heat and Mass Transfer MHD Flow of Micropolar Fluid past a Vertical Porous Plate in a Porous Medium
}

\author{
Parash Moni Thakur \\ Department of Mathematics \\ D. K. D. College, Deragaon \\ Assam, India
}

\author{
G.C. Hazarika \\ Dibrugarh University \\ Dibrugarh \\ Assam, India
}

\begin{abstract}
The effect of temperature dependent viscosity and thermal conductivity on combined free-forced convection heat and mass transfer MHD flow of micropolar fluid past a vertical porous plate in a porous medium is studied where the viscosity and thermal conductivity are assumed to be inverse linear functions of temperature. The partial differential equations governing the flow with heat and mass transfer of the problem are transformed into dimensionless form of ordinary differential equations by using similarity substitutions. The governing boundary value problems are then solved numerically using shooting method. The effects of various parameters viz. viscosity parameter, thermal conductivity parameter, thermal buoyancy parameter, mass buoyancy parameter, coupling constant parameter, Prandtl number and magnetic parameter on velocity, micro-rotation, temperature and species concentration fields are investigated and presented graphically. The coefficient of skin-friction, Nusselt number and Sherwood number are also computed and presented tabular form.
\end{abstract}

\section{Keywords}

Micropolar fluid, variable viscosity, thermal conductivity, heat and mass transfer, MHD Flow, porous medium.

\section{INTRODUCTION}

The concept of micropolar fluid has been derived from the need to model the flow of fluids that contain rotating microconstituents; see [2] - [4]. In theory of micropolar fluid, each particle has a finite size and constitutes a micro structure which can rotate about the centre of the volume element described by the micro-rotation vector. The effects of magneto hydrodynamics on the flow of micropolar fluid has become important due to several engineering applications such as in MHD generators, designing cooling system for nuclear reactor, flow meters etc., where the micro concentration provides an important parameter for deciding the rate of heat flow. By simulating it one can obtain the desired temperature in such equipments. Several investigators have made theoretical and experimental studies of micropolar flow in the presence of a transverse magnetic field during the last few decades. Assuming fluid viscosity as a linear function of temperature the effect of variable viscosity on MHD natural convection in micropolar fluids was studied by Abd El-Hakiem et al. [1]. Gorla et al. [5] investigated the Magnetohydrodynamic free convection boundary layer flow of a thermo micropolar fluid over a vertical plate. Gorla [6] studied the flow of a micropolar fluid over a flat plate. Ishak et al. [8] studied the problem of steady boundary layer flow and heat transfer of a micropolar fluid on an isothermal continuously moving plane surface assuming that the microinertia density is variable and not constant. Modather et al. [10] studied variable viscosity effect on heat transfer over a continuous moving surface with variable internal heat generation in micropolar fluids. Peddieson and Mecnitt [11] investigated boundary layer theory for micro polar fluid. Rajesh [12] investigated the MHD free convection flow past an accelerated vertical porous plate with variable temperature through a porous medium. Salem and Odda [13] studied influence of variable viscosity and thermal conductivity on flow of micropolar fluid past a continuously moving plate with suction or injection. Sharma and Hazarika [14] studied the effects of variable viscosity and thermal conductivity on heat and mass transfer flow of Newtonian fluid along a vertical plate in the presence magnetic field. They [15] investigated effects of variable viscosity and thermal conductivity on combined free-forced convection and mass transfer flow passed a vertical porous plate.

The main objective of the present work is to extend the work of Sharma and Hazarika [15] to study the effects of variable viscosity and thermal conductivity on combined free-forced convection and mass transfer flow passed a vertical porous plate in porous medium. Viscosity and thermal conductivity are assumed to be inverse linear functions of temperature. The governing partial differential equations are reduced in to ordinary differential equations by similarity transformations. The problem is then solved numerically using Runge-Kutta shooting algorithm with iteration process.

\section{MATHEMATICAL FORMULATION OF THE PROBLEM:}

A steady free-forced convective flow of a viscous incompressible micropolar electrically conducting fluid past a semi-infinite vertical plate, at constant temperature $T_{w}$, in the presence of uniform transverse magnetic field is considered. The temperature of the fluid far from the plate is $T_{\infty}$. The $x$-axis is taken along the plate in upward direction and the $y$-axis is taken normal to it. The plate is parallel to the direction which is also the direction of gravity but directed vertically upward. Also a magnetic field of constant intensity is assumed to be applied normal to the vertical plate and the electrical conductivity of the fluid is assumed to be so small that the induced magnetic field can be neglected in comparison to the applied magnetic field. The applied magnetic field is primary in the $y$-direction and is a function of $x$ only. $(u(y), 0)$ are the velocity components and $N$ is the component of micro-rotation perpendicular to the $x y$-plane.The free stream velocity $U_{\infty}$, parallel to the vertical 
plate, is assumed to be constant Under these assumptions the governing equations of the problem are:

\subsection{Basic Equations:}

\section{Equation of continuity:}

$$
\frac{\partial u}{\partial x}+\frac{\partial v}{\partial y}=0
$$

\section{Momentum equations:}

$$
\begin{aligned}
& \frac{\partial u}{\partial t}+v \frac{\partial u}{\partial y}=g_{0} \beta\left(T-T_{\infty}\right)+g_{0} \beta^{*}\left(C-C_{\infty}\right) \\
& +\frac{\partial}{\partial y}\left(v \frac{\partial u}{\partial y}\right)+\frac{\kappa}{\rho}\left(\frac{\partial N}{\partial y}+\frac{\partial^{2} u}{\partial y^{2}}\right)-\frac{v u}{K^{\prime}}-\frac{\sigma B^{2}}{\rho} u
\end{aligned}
$$

\section{Angular momentum equation:}

$$
\frac{\partial N}{\partial t}+v \frac{\partial N}{\partial y}=\frac{\gamma}{\rho \mathrm{j}} \frac{\partial^{2} N}{\partial y^{2}}-\frac{\kappa}{\rho \mathrm{j}}\left(2 N+\frac{\partial u}{\partial y}\right)
$$

\section{Energy equation:}

$\frac{\partial T}{\partial t}+v \frac{\partial T}{\partial y}=\frac{1}{\rho c_{\mathrm{p}}} \frac{\partial}{\partial y}\left(\lambda \frac{\partial T}{\partial y}\right)+\frac{Q_{0}}{\rho c_{p}}\left(T-T_{\infty}\right)$

\section{Species continuity equation:}

$$
u \frac{\partial C}{\partial x}+v \frac{\partial C}{\partial y}=\frac{1}{\mathrm{~S}_{\mathrm{c}}} \frac{\partial}{\partial y}\left(v \frac{\partial C}{\partial y}\right)+D_{T} \frac{\partial^{2} T}{\partial y^{2}}+D_{T} \frac{\partial v}{\partial y} \frac{\partial T}{\partial y}
$$

The equation of continuity is being identically satisfied by velocity profile $(u(y), 0)$. It should be mentioned that $N=0$ indicates strong concentration and represents concentrated particles flows in which the microelements close to the wall surface are unable to translate or rotate. It is known (Gorla [5]) that $N$ is the total spin of microstructure and fluid media in the flow field. In some cases, the microstructure effects become negligible and the fluid behaves like an ordinary (Newtonian) viscous flow. Therefore, if we state that $N=$ angular velocity is a valid solution, then this is possible only if

$\gamma=\left(\mu_{\infty}+\frac{\kappa}{2}\right) j=\mu_{\infty}\left(1+\frac{K_{1}}{2}\right) j$, where $K_{1}$ is the coupling constant parameter.

Lai and Kulacki [9] have assumed that the viscosity is an inverse linear function of temperature, i.e.

$\frac{1}{\mu}=\frac{1}{\mu_{\infty}}\left[1+\delta\left(T-T_{\infty}\right)\right]$, or $\frac{1}{\mu}=a\left(T-T_{c}\right)$ where $a=$

$\frac{\delta}{\mu_{\infty}}$ and $T_{c}=T_{\infty}-\frac{1}{\delta}$

Following Lai and Kulaski, Hazarika and Khound [7] assumed the thermal conductivity as

$$
\begin{aligned}
& \frac{1}{\lambda}=\frac{1}{\lambda_{\infty}}\left[1+\xi\left(T-T_{\infty}\right)\right], \text { or } \frac{1}{\lambda}=b\left(T-T_{r}\right) \\
& \text { where } b=\frac{\xi}{\lambda_{\infty}} \text { and } T_{r}=T_{\infty}-\frac{1}{\xi} .
\end{aligned}
$$

Here $\mu$ is the viscosity of the fluid, $\mu_{\infty}$ is the viscosity of the fluid at infinity, $T$ is the fluid temperature, $T_{\infty}$ is the temperature of the free stream, $\lambda$ and $\lambda_{\infty}$ are the thermal conductivities at temperatures $T$ and $T_{\infty}$ respectively; $a, b, T_{c}$ and $T_{r}$ are constants based on the reference state and the thermal property of the fluid.

The appropriate boundary conditions are

$$
\left.\begin{array}{l}
y=0: u=0, v=v_{w}, N=0, T=T_{w}, C=C_{w} \\
y \rightarrow \infty: u=U_{\infty}, T=T_{\infty}, \quad C=C_{\infty}, N=0
\end{array}\right\}
$$

We introduce the following non-dimensional variables:

$$
\left.\begin{array}{c}
\eta=y \sqrt{\frac{U_{\infty}}{v_{\infty} x}}, \psi=\sqrt{U_{\infty} v_{\infty} x} f(\eta), \frac{T-T_{\infty}}{T_{w}-T_{\infty}}=\theta(\eta), \\
g(\eta)=\frac{C-C_{\infty}}{C_{w}-C_{\infty}}, N=\sqrt{\frac{U_{\infty}^{3}}{v_{\infty} x}} h(\eta), B=B_{0} x^{-\frac{1}{2}}, j=\frac{\nu_{\infty} x}{U_{\infty}^{2}} i(\eta)
\end{array}\right\} .
$$

Substituting above transformations in equations (2) - (5), we get

$$
\begin{gathered}
\left(1+K_{1} \frac{\theta_{c}-\theta}{\theta_{c}}\right) f^{\prime \prime \prime}=\left[g_{t} \theta+g_{m} g+K_{1} h^{\prime}-M f^{\prime}+\frac{1}{2} f f^{\prime \prime}\right] \frac{\theta-\theta_{c}}{\theta_{c}}+ \\
K f^{\prime}+\frac{\theta^{\prime} f^{\prime \prime}}{\theta-\theta_{c}} \\
\left(1+\frac{1}{2} K_{1}\right) i h^{\prime \prime}=\left(h f^{\prime}+h^{\prime} f\right) i-K_{1}\left(2 h+f^{\prime \prime}\right) \\
\theta^{\prime \prime}=P_{r}\left(\frac{1}{2} f \theta^{\prime}+Q \theta\right) \frac{\theta-\theta_{r}}{\theta_{r}}+\frac{\theta^{\prime 2}}{\theta-\theta_{r}} \\
g^{\prime \prime}=S_{c}\left[\frac{1}{2} f g^{\prime}+S_{r}\left\{P_{r}\left(\frac{1}{2} f \theta^{\prime}+Q \theta\right) \frac{\theta-\theta_{r}}{\theta_{r}}+\frac{\theta^{\prime 2}}{\theta-\theta_{r}}\right\}\right] \frac{\theta-\theta_{c}}{\theta_{c}}+ \\
\frac{g^{\prime} \theta^{\prime}}{\theta-\theta_{c}}+S_{c} S_{r} \frac{\theta^{\prime 2}}{\theta-\theta_{c}}
\end{gathered}
$$

The boundary conditions (6) become

$$
\left.\begin{array}{c}
\eta=0: f=f_{w}, f^{\prime}=0, g=1, \theta=1, h=0 \\
\eta \rightarrow \infty: f^{\prime}=1, g=0, \theta=0, h=0
\end{array}\right\}
$$

The dimensionless parameters are defined as follows:

$$
\begin{aligned}
& f_{w}=-2 v_{w} \sqrt{\frac{x}{v_{\infty} U_{\infty}}}, K_{1}=\frac{\kappa}{v_{\infty} \rho}, K=\frac{v_{\infty} x}{U_{\infty} \mathrm{K}^{\prime}}, M=\frac{\sigma B_{0}{ }^{2}}{U_{\infty} \rho}, P_{r}= \\
& \frac{v_{\infty} \rho c_{p}}{\lambda_{\infty}}, Q=\frac{Q_{0} x}{v_{\infty} \rho c_{p}}, R_{e}=\frac{U_{\infty} x}{v_{\infty}}, g_{t}=\frac{G_{r}}{R_{e}{ }^{2}}, g_{m}=\frac{G_{c}}{R_{e}{ }^{2}}, S_{c}= \\
& \frac{v_{\infty}}{D}, G_{r}=\frac{g_{0} \beta\left(T_{w}-T_{\infty}\right) x}{U_{\infty}^{2}}, G_{c}=\frac{g_{0} \beta^{*}\left(C_{w}-C_{\infty}\right) x}{U_{\infty}^{2}}, S_{r}=\frac{D_{T}\left(T_{w}-T_{\infty}\right)}{v_{\infty}\left(C_{w}-C_{\infty}\right)},
\end{aligned}
$$

The physical quantities of interest in this problem are the skin-friction coefficient $c_{f}$, Nusselt number $N u$ and Sherwood number $S_{h}$ which indicate physically wall shear stress, rate of heat transfer and rate of mass transfer respectively. For micropolar boundary layer flow, the wall shear stress $\tau_{w}$ is given by

$$
\tau_{w}=\left[(\mu+k) \frac{\partial u}{\partial y}+k N\right]_{y=0}
$$

The skin-friction coefficient $c_{f}$ can be defined as

$$
c_{f}=\frac{2 \tau_{w}}{\rho U_{\infty}^{2}}=2\left(\frac{\theta_{c}}{\theta_{c}-1}+K_{1}\right) R_{e^{-\frac{1}{2}} f^{\prime \prime}(0)}
$$

The heat transfer from the plate is given by 
$q_{w}=-\lambda\left[\frac{\partial T}{\partial y}\right]_{y=0}=\lambda_{\infty} \frac{\theta_{r}}{1-\theta_{r}}\left[\frac{\partial T}{\partial y}\right]_{y=0}$

$N u=\frac{x q_{w}}{\lambda_{\infty}\left(T_{w}-T_{\infty}\right)}=\frac{\theta_{r}}{1-\theta_{r}} R_{e}^{\frac{1}{2}} \theta^{\prime}(0)$

The mass flux at the wall is given by

$$
\begin{aligned}
M_{w} & =-D\left[\frac{\partial C}{\partial y}\right]_{y=0} \\
S_{h} & =\frac{S_{c} M_{w} x}{v_{\infty}\left(C_{w}-C_{\infty}\right)}=\frac{\theta_{c}}{1-\theta_{c}} R_{e}^{\frac{1}{2}} g^{\prime}(0)
\end{aligned}
$$

\section{RESULTS AND DISCUSSIONS}

The equations (8) - (11) together with the boundary conditions (12) are solved for various combinations of the parameters involved in the equations using an algorithm based on the shooting method and results are presented graphically for the dimensionless velocity, micro-rotation, species concentration and temperature distributions with the variation of different parameters.

The values of different parameters have been taken as $g_{t}=0.1$, $g_{m}=0.5, M=1, \operatorname{Pr}=0.7, K_{1}=0.1 \mathrm{~K}=0.1, Q=0.1$ and $S_{c}=1$, $S_{r}=0.1$ with the viscosity parameter $\theta_{c}$ ranging from -10 to 1 at certain value of $\theta_{r}=-10$ unless otherwise stated. Similarly solutions have been found with varying the thermal conductivity parameter $\theta_{r}$ ranging from -10 to -1 at certain value of $\theta_{c}=-10$ keeping the other values unchanged. Solutions have also been found for different values of magnetic parameter $(M)$, temperature buoyancy parameter $\left(g_{t}\right)$, mass buoyancy parameter $\left(g_{m}\right)$, dimensionless reference temperature corresponding to thermal conductivity parameter $\left(\theta_{r}\right)$, dimensionless reference temperature corresponding to viscosity parameter $\left(\theta_{c}\right)$, Prandtl number $\left(P_{r}\right)$, the coupling constant parameter $\left(K_{1}\right)$, Permeability parameter $(K)$, local heat generation parameter $(\mathrm{Q})$, Soret number $\left(S_{r}\right)$ and Schmidt number $(\mathrm{Sc})$.

The variations in dimensionless velocity, temperature, species concentration and micro-rotation distributions are illustrated in figures (1) - (14) with the variation of different parameters.

\subsection{Figures}

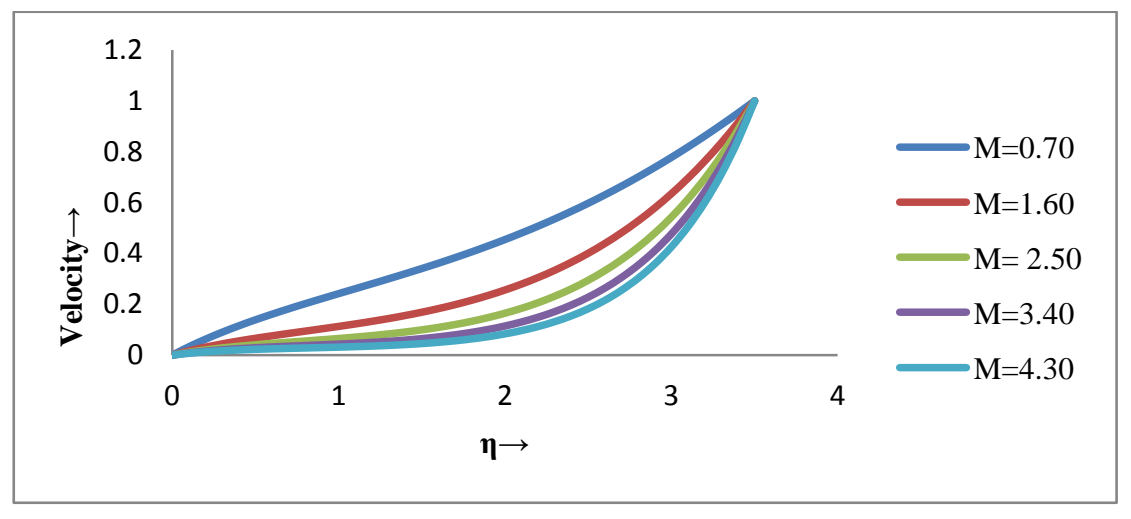

Figure 1 Velocity distributions against $\eta$ for different values of $M$ 


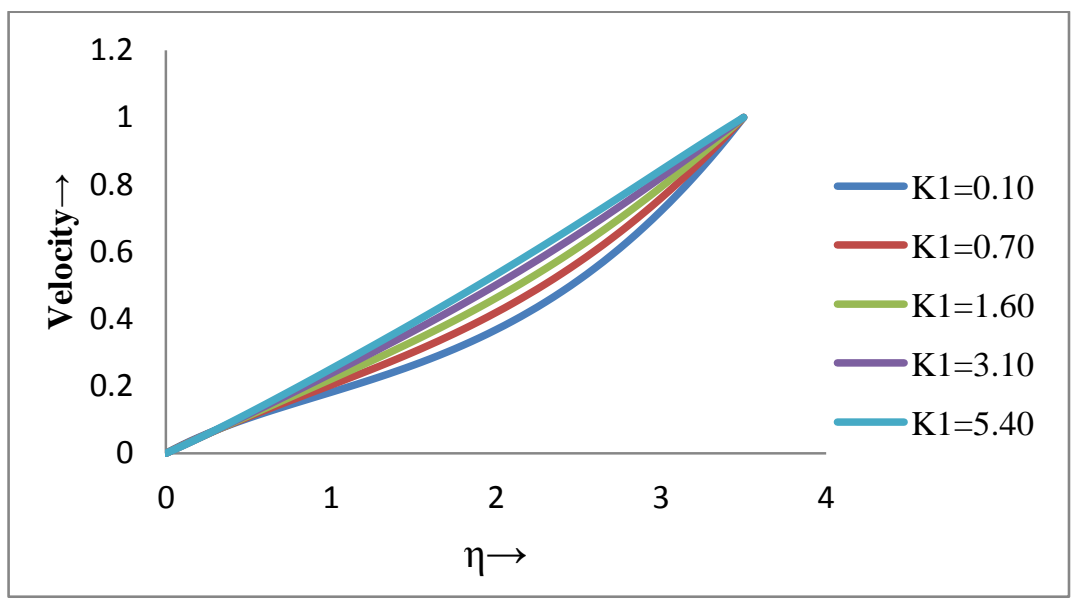

Figure 2 Velocity distributions against $\boldsymbol{\eta}$ for different values of $K_{1}$

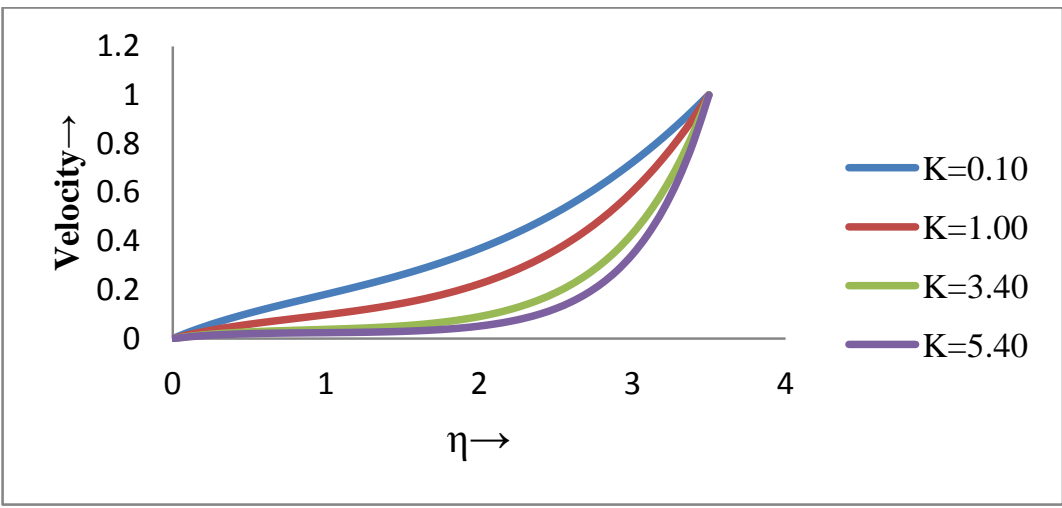

Figure 3 Velocity distributions against $\eta$ for different values of $K$

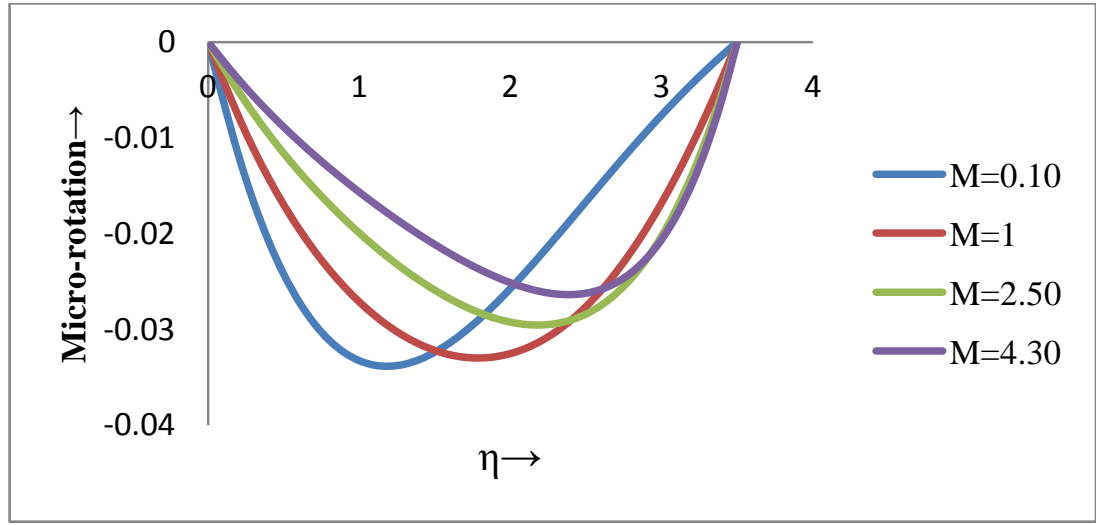

Figure 4 Micro-rotation distributions against $\eta$ for different values of $\boldsymbol{M}$ 


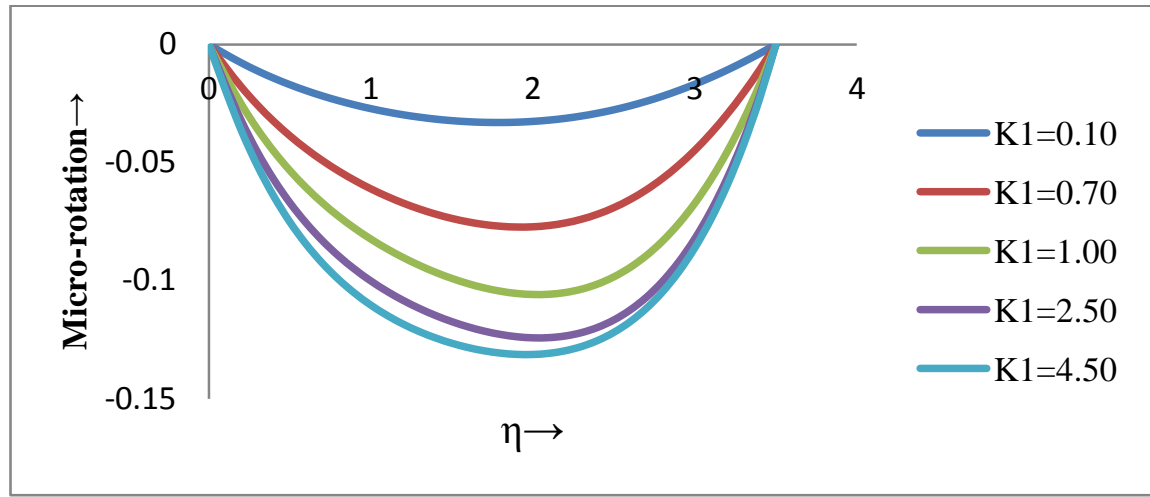

Figure 5 Micro-rotation distributions against $\eta$ for different values of $K_{1}$

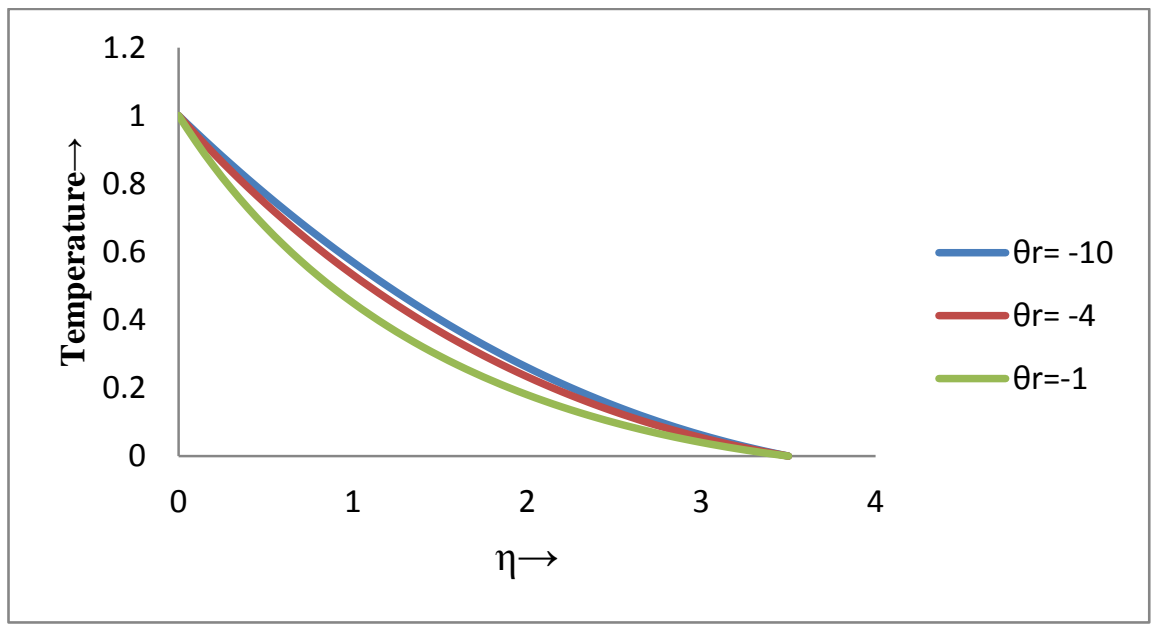

Figure 6 Temperature distributions against $\eta$ for different values of $\theta_{r}$

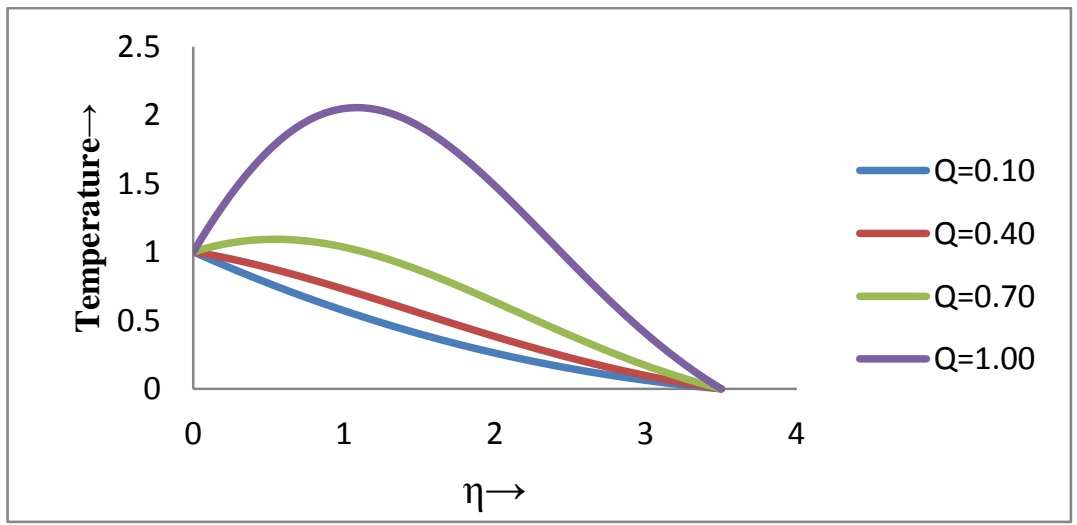

Figure 7 Temperature distributions against $\eta$ for different values of $Q$ 


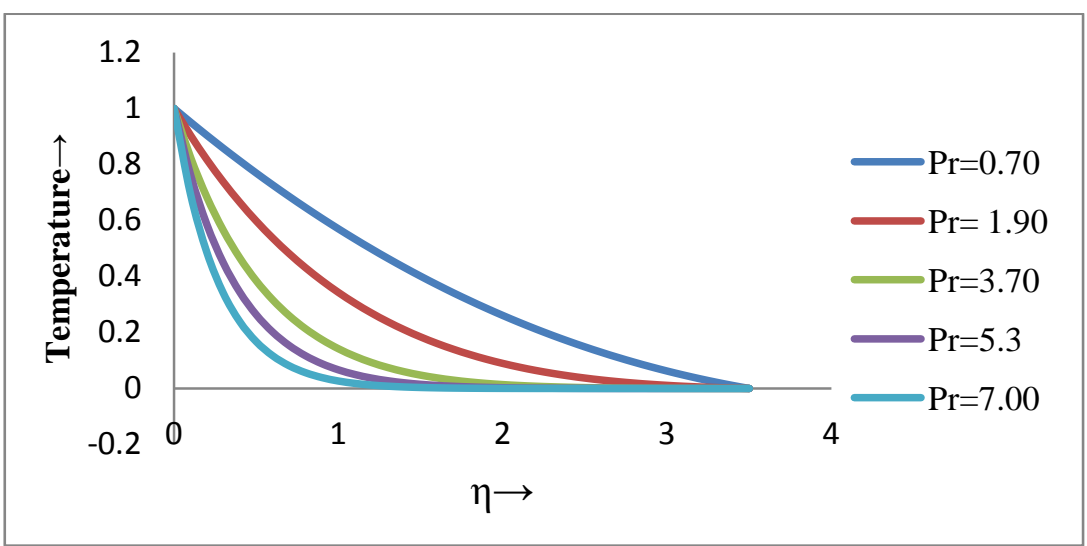

Figure 8 Temperature distributions against $\eta$ for different values of $P_{r}$

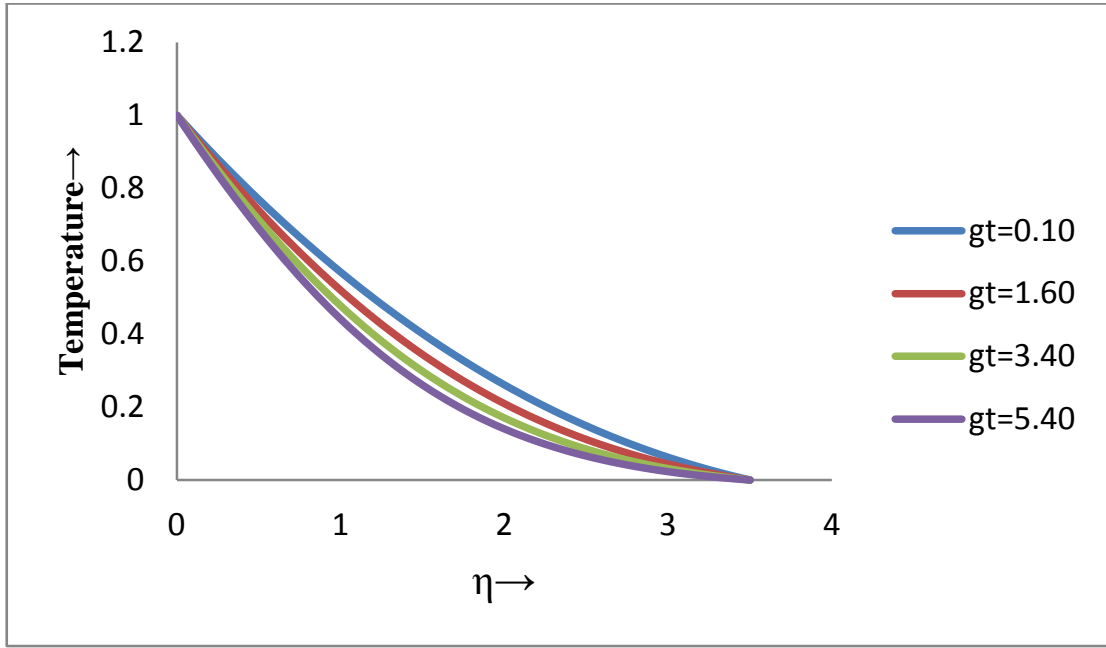

Figure 9 Temperature distributions against $\eta$ for different values of $g_{t}$

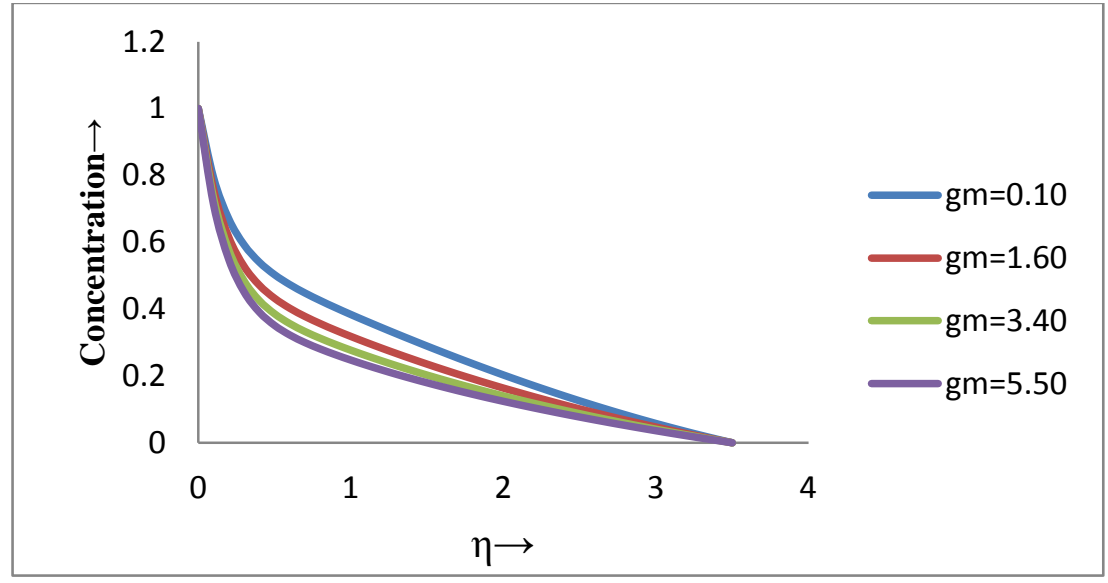

Figure 10 Concentration distributions against $\boldsymbol{\eta}$ for different values of $g_{m}$ 


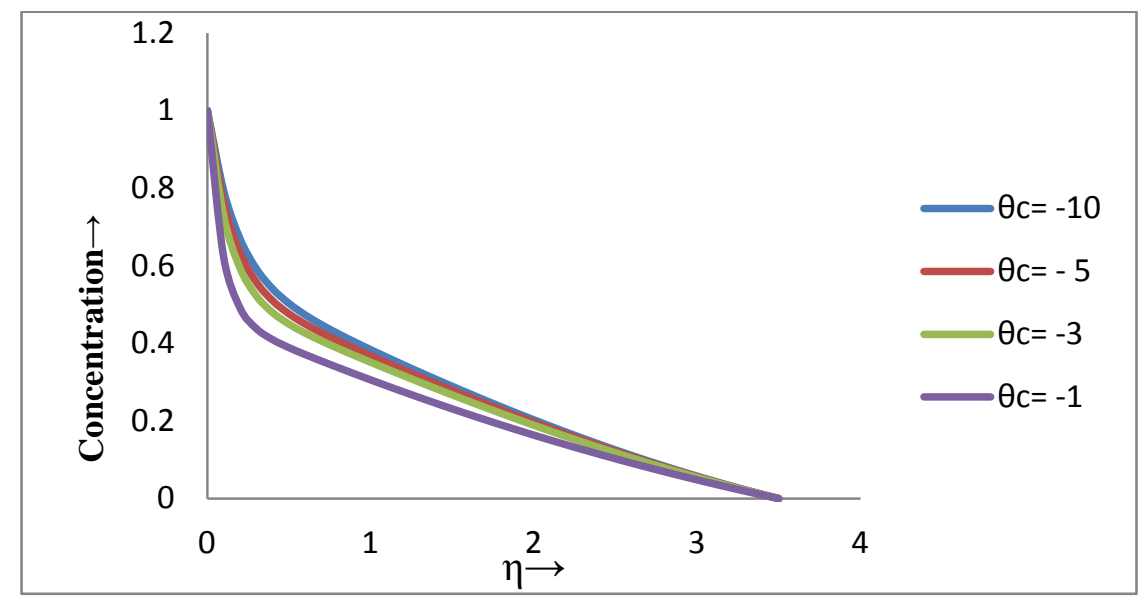

Figure 11 Concentration distributions against $\boldsymbol{\eta}$ for different values of $\boldsymbol{\theta}_{c}$

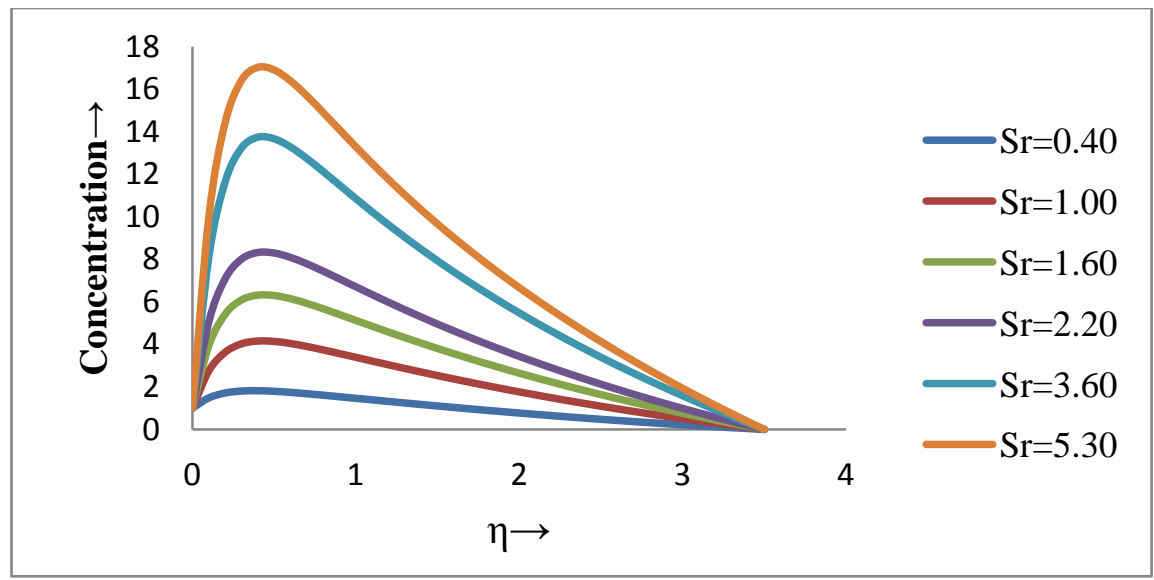

Figure 12 Concentration distributions against $\eta$ for different values of $S_{r}$

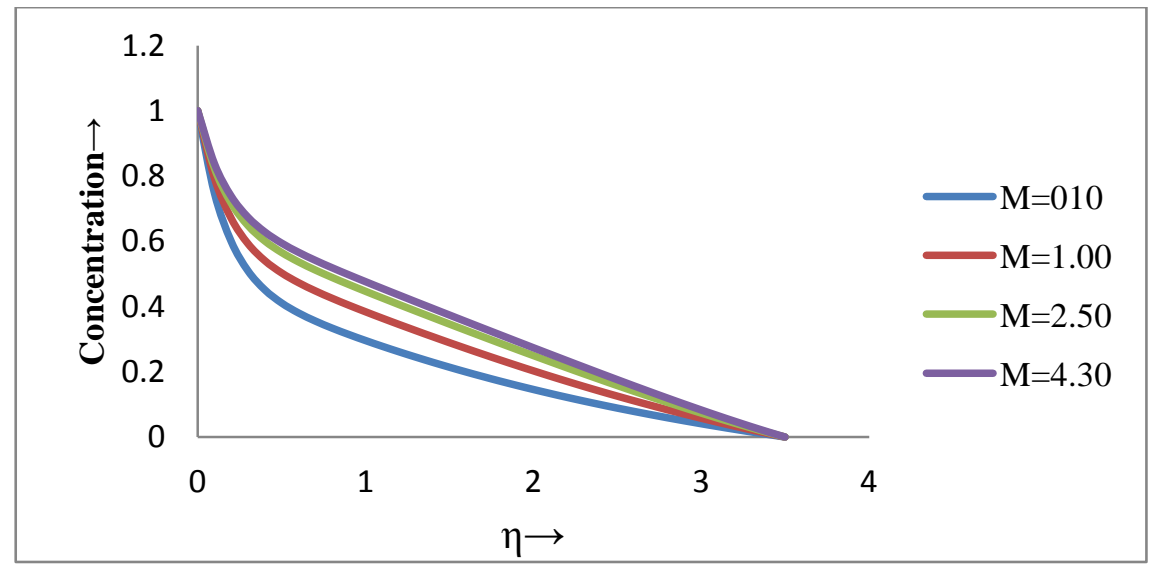

Figure 13 Concentration distributions against $\boldsymbol{\eta}$ for different values of $\boldsymbol{M}$ 


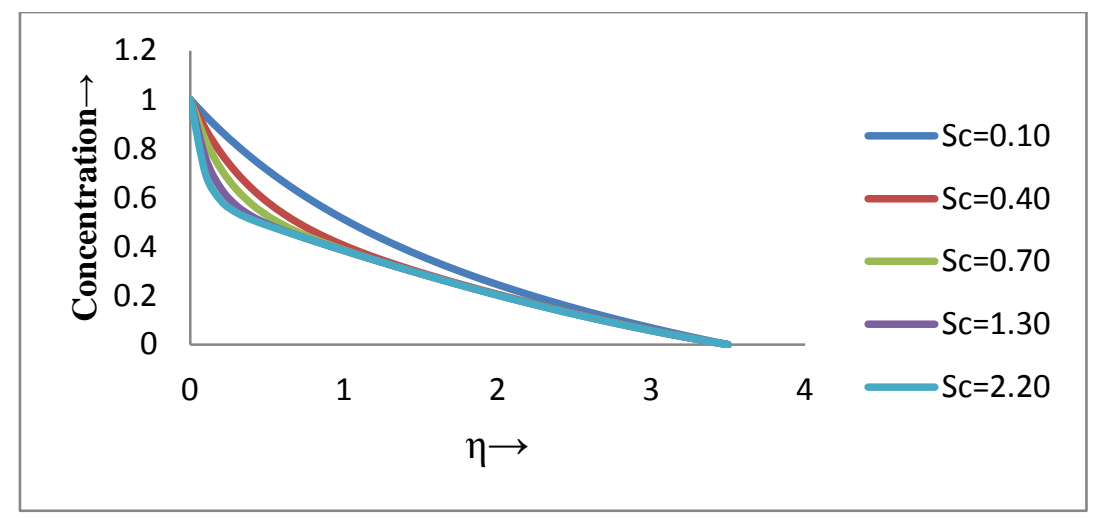

Figure 14 Concentration distributions against $\boldsymbol{\eta}$ for different values of $\boldsymbol{S}_{c}$

\subsection{Tables}

Finally the effects of the above mentioned parameters on the values of $f^{\prime}(0), g^{\prime}(0), h^{\prime}(0), \theta^{\prime}(0), c_{f}, N u$ and $S_{h}$ are shown in the tables (1) - (4). We consider $\theta_{c}=-10, \theta_{r}=-10, g_{t}=0.1, g_{m}=0.5, M=1, \operatorname{Pr}=0.7, K_{1}=0.1 \mathrm{~K}=0.1, Q=0.1$ and $S_{c}=1, S_{r}=0.1$.The behavior of these parameters is self evident from the tables and hence any further discussions about them seem to be redundant.

Table 1: Missing values and physical quantities with the variation of $\operatorname{Pr}$

\begin{tabular}{|c|c|c|c|c|c|c|c|}
\hline $\operatorname{Pr}$ & $f^{\prime}(0)$ & $g^{\prime}(0)$ & $h^{\prime}(0)$ & $\theta^{\prime}(0)$ & $c_{f}$ & $N u$ & $S_{h}$ \\
\hline 0.5 & 0.271387 & -2.65868 & -0.0399 & -0.42892 & 1.732003 & 0.123306 & 0.764315 \\
\hline 1.5 & 0.258027 & -2.78714 & -0.03932 & -0.81575 & 1.646743 & 0.23451 & 0.801247 \\
\hline 2.5 & 0.247654 & -2.92867 & -0.03897 & -1.29607 & 1.58054 & 0.372594 & 0.841932 \\
\hline 3.5 & 0.240362 & -3.06692 & -0.03879 & -1.81151 & 1.534 & 0.520771 & 0.881678 \\
\hline 4.5 & 0.235182 & -3.19832 & -0.03869 & -2.34103 & 1.500943 & 0.672998 & 0.919453 \\
\hline 5.5 & 0.231372 & -3.32288 & -0.03863 & -2.87751 & 1.476627 & 0.827227 & 0.955262 \\
\hline 6.5 & 0.228472 & -3.44139 & -0.03859 & -3.41802 & 1.458123 & 0.982612 & 0.989329 \\
\hline 7.5 & 0.226202 & -3.55479 & -0.03857 & -3.9611 & 1.443633 & 1.138736 & 1.02193 \\
\hline 8.5 & 0.224382 & -3.66415 & -0.03856 & -4.50597 & 1.432016 & 1.295376 & 1.053369 \\
\hline
\end{tabular}

Table 2: Missing values and physical quantities with the variation of $\theta_{c}$

\begin{tabular}{|c|c|c|c|c|c|c|c|}
\hline$\theta_{c}$ & $f^{\prime}(0)$ & $g^{\prime}(0)$ & $h^{\prime}(0)$ & $\theta^{\prime}(0)$ & $c_{f}$ & $N u$ & $S_{h}$ \\
\hline-10 & 0.268605 & -2.681801 & -0.039765 & -0.494309 & 1.71425 & 0.142104 & 0.770964 \\
\hline-9 & 0.269785 & -2.740596 & -0.039776 & -0.494368 & 1.706272 & 0.142121 & 0.779987 \\
\hline-8 & 0.271248 & -2.813696 & -0.039789 & -0.494441 & 1.696465 & 0.142142 & 0.790906 \\
\hline-7 & 0.27311 & -2.907036 & -0.039806 & -0.494533 & 1.684116 & 0.142168 & 0.804375 \\
\hline-6 & 0.275558 & -3.030352 & -0.039827 & -0.494653 & 1.668091 & 0.142203 & 0.821384 \\
\hline-5 & 0.278922 & -3.200805 & -0.039856 & -0.494816 & 1.646454 & 0.14225 & 0.843486 \\
\hline-4 & 0.283835 & -3.451737 & -0.039898 & -0.49505 & 1.615619 & 0.142317 & 0.873228 \\
\hline-3 & 0.291691 & -3.857649 & -0.039962 & -0.495412 & 1.568095 & 0.142421 & 0.914922 \\
\hline-2 & 0.306286 & -4.626873 & -0.040069 & -0.496046 & 1.485128 & 0.142603 & 0.975431 \\
\hline-1 & 0.343138 & -6.668235 & -0.040276 & -0.497393 & 1.302118 & 0.14299 & 1.054341 \\
\hline
\end{tabular}


Table 3: Missing values and physical quantities with the variation of $\theta_{r}$

\begin{tabular}{|c|c|c|c|c|c|c|c|}
\hline$\theta_{r}$ & $f^{\prime}(0)$ & $g^{\prime}(0)$ & $h^{\prime}(0)$ & $\theta^{\prime}(0)$ & $c_{f}$ & $N u$ & $S_{h}$ \\
\hline-10 & 0.268605 & -2.6818 & -0.03977 & -0.49431 & 1.71425 & 0.142104 & 0.770964 \\
\hline-9 & 0.268478 & -2.68305 & -0.03976 & -0.4983 & 1.71344 & 0.141819 & 0.771322 \\
\hline-8 & 0.26832 & -2.6846 & -0.03975 & -0.50329 & 1.712435 & 0.141469 & 0.771768 \\
\hline-7 & 0.26812 & -2.68659 & -0.03975 & -0.50968 & 1.711157 & 0.141029 & 0.772339 \\
\hline-6 & 0.267857 & -2.68922 & -0.03974 & -0.5182 & 1.709478 & 0.140459 & 0.773096 \\
\hline-5 & 0.267495 & -2.69288 & -0.03972 & -0.53009 & 1.707169 & 0.13969 & 0.774147 \\
\hline-4 & 0.266967 & -2.6983 & -0.0397 & -0.54786 & 1.703799 & 0.138599 & 0.775708 \\
\hline-3 & 0.266123 & -2.7072 & -0.03967 & -0.57733 & 1.698409 & 0.136925 & 0.778265 \\
\hline-2 & 0.264552 & -2.72448 & -0.03961 & -0.63579 & 1.688385 & 0.134036 & 0.783233 \\
\hline-1 & 0.26057 & -2.77306 & -0.03946 & -0.80854 & 1.662973 & 0.127841 & 0.797198 \\
\hline
\end{tabular}

Table 4: Missing values and physical quantities with the variation of $Q$

\begin{tabular}{|c|c|c|c|c|c|c|c|}
\hline$Q$ & $f^{\prime}(0)$ & $g^{\prime}(0)$ & $h^{\prime}(0)$ & $\theta^{\prime}(0)$ & $c_{f}$ & $N u$ & $S_{h}$ \\
\hline 0.1 & 0.268605 & -2.6818 & -0.03977 & -0.49431 & 1.71425 & 0.142104 & 0.770964 \\
\hline 0.2 & 0.271451 & -2.65404 & -0.03988 & -0.40416 & 1.732414 & 0.116187 & 0.762983 \\
\hline 0.3 & 0.274818 & -2.62264 & -0.04002 & -0.30194 & 1.753905 & 0.086801 & 0.753957 \\
\hline 0.4 & 0.278883 & -2.5866 & -0.04019 & -0.18376 & 1.779843 & 0.052827 & 0.743594 \\
\hline 0.5 & 0.283911 & -2.54444 & -0.0404 & -0.04372 & 1.811936 & 0.012569 & 0.731474 \\
\hline 0.6 & 0.290334 & -2.49401 & -0.04068 & 0.127592 & 1.852924 & -0.03668 & 0.716976 \\
\hline 0.7 & 0.298889 & -2.43198 & -0.04105 & 0.346288 & 1.907526 & -0.09955 & 0.699144 \\
\hline 0.8 & 0.310976 & -2.35297 & -0.04158 & 0.64263 & 1.984664 & -0.18474 & 0.676431 \\
\hline 0.9 & 0.329623 & -2.24797 & -0.0424 & 1.081645 & 2.103673 & -0.31095 & 0.646246 \\
\hline
\end{tabular}

\section{CONCLUSION}

In this study the effects of variable viscosity and thermal conductivity on combined free-forced convection and mass transfer flow passed a vertical porous plate in porous medium in presence of applied magnetic field are investigated. The results demonstrate clearly that the viscosity and thermal conductivity parameters along with the other parameters mentioned above have significant effects on velocity, temperature, concentration and micro-rotation distributions within the boundary layer. Thus presence of microconstituents results significant behaviours in the flow problems in micropolar fluid in comparison with the ordinary fluids.

\section{NOMENCLATURES}

$\beta=$ Volumetric coefficient of thermal expansion

$\beta^{*}=$ Volumetric co-efficient of expansion with concentration

$g_{0}=$ Gravitational acceleration

$\lambda=$ Thermal conductivity

$\mu=$ Dynamic viscosity

$\mu_{\infty}=$ Dynamic viscosity of the ambient fluid $v=$ Kinematic viscosity

$v_{\infty}=$ Kinematic Viscosity of the ambient fluid

$\kappa=$ Vortex viscosity

$\gamma=$ Spin-gradient or micro rotation viscosity

$\mathrm{c}_{\mathrm{p}}=$ Specific heat

$\mathrm{f}_{\mathrm{w}}=$ Suction parameter

$\eta=$ Dimensionless co-ordinates

$u=$ Velocity in the $x$-direction

$v=$ Velocity in the $y$-direction

$w=$ Secondary velocity

$U_{\infty}=$ Reference velocity

$f=$ Dimensionless velocity

$h=$ Dimensionless micro-rotation

$g=$ Dimensionless species concentration

$\theta=$ Dimensionless temperature 
$\theta_{c}=$ Dimensionless reference temperature corresponding to viscosity parameter

$\theta_{r}=$ Dimensionless reference temperature corresponding to thermal conductivity parameter

$T=$ Temperature

$C=$ Species concentration

$T_{\infty}=$ Ambient temperature

$T_{w}=$ Wall temperature

$C_{w}=$ Species concentration at the wall

$C_{\infty}=$ Species concentration far from the wall

$j=$ Micro-inertia density

$\sigma=$ Electrical conductivity

$\rho=$ Density

$D=$ Thermal molecular diffusivity

$g_{t}=$ Temperature buoyancy parameter

$G_{C}=$ Mass buoyancy parameter

$S_{c}=$ Schmidt number

$P_{r}=$ Prandtl number

$R_{e}=$ Local Reynolds number

$M=$ Hartmann Number

$Q=$ local heat generation parameter

$B_{0}=$ Constant magnetic field intensity

$K=$ Permeability parameter

$K_{1}=$ Coupling constant parameter

$q_{w}=$ Heat transfer from the plate

$c_{\mathrm{f}}=$ Skin-friction coefficient

$N u=$ Nusselt number

$S_{h}=$ Sherwood number

\section{Subscripts:}

$\mathbf{w}$, the condition at the wall

$\infty$, the condition far away from the surface

\section{Superscripts:}

${ }^{g}$, Differentiation with respect to $\eta$

\section{REFERENCES}

[1] Abd El-hakiem M., Modather M., Abdou M, The effect of variable viscosity on MHD natural convection in micropolar fluids, Int. J. Appl. Mech. Engg., Vol.11,No. 2, pp. 301-320(2006).
[2] Eringen A.C., Simple micro fluids, Int. J. Eng. Sci., Vol.2, pp. 205-217(1964)

[3] Eringen A.C., Theory of micropolar fluids, J. Math. Mech., Vol.16, pp.1-18 (1966).

[4] Eringen A.C., Theory of Thermomicropolar fluids, J. Math. Anal. Appl., Vol.38, pp.480- 496 (1972).

[5] Gorla R.S.R., Takhar H.S., Slaouti A., Magneto hydrodynamic free convection boundary layer flow of a thermo micropolar fluid over a vertical plate, Int. J. Eng. Sci., Vol. 36, pp. 315-327(1998).

[6] Gorla R.S., Heat transfer in micropolar boundary layer flow over a flat plate, Int. J. Eng. Sci., Vol. 21, pp. $791-$ 796(1983).

[7] Hazarika G.C. and Khound P.K., The effect of variable viscosity and thermal conductivity on liquid film on an unsteady stretching surface, Proc. of $46^{\text {th }}$ Annual Tech. Session, Ass. Sc. Soc., pp.47-56 (2000).

[8] Ishak A., Nazar R., Pop I., Flow of a micropolar fluid on a continuous moving surface, Arch. Mech., Vol.58, No.6, pp.529-541(2006).

[9] Lai, F.C. and Kulacki, F.A., The effect of variable viscosity on convective heat and mass transfer along a vertical surface in saturated porous medium, Int. J.Heat and Mass Transfer, Vol.33, 1028-1031.

[10] Modather M., Abdou M., Roshdy El-Zahar E., Variable viscosity effect on heat transfer over a continuous moving surface with variable internal heat generation in micropolar fluids, Appl. Math. Sci.,Vol. 6, No.128, pp. 6365-6379 (2012).

[11] Peddieson J., Mecnitt R. P., Boundary layer theory for a micropolar fluid, Recent Adv. Engng. Sci.,Vol.5 pp.405425 (1997).

[12] Rajesh V., MHD free convection flow past an accelerated vertical porous plate with variable temperature through a porous medium, Acta Technica Corviniensis, Bulletin and Engineering, pp.91-96

[13] Salem A. M., Odda S. N., Influence of variable viscosity and thermal conductivity on flow of micropolar fluid past a continuously moving plate with suction or injection, The Korean society for Ind. Appl. Math., 2005

[14] Sarma U., Hazarika G. C., Effects of variable viscosity and thermal conductivity on heat and mass transfer flow along a vertical plate in the presence magnetic field, Lat. Am. J. Phys. Educ. Vol.5, No.1, 2011.

[15] Sarma U., Hazarika G. C. Effects of variable viscosity and thermal conductivity on combined free-forced convection and mass transfer flow passed a vertical porous plate, Antarctica J. Math., Vol.7,No.4, pp.461$472,2010$. 